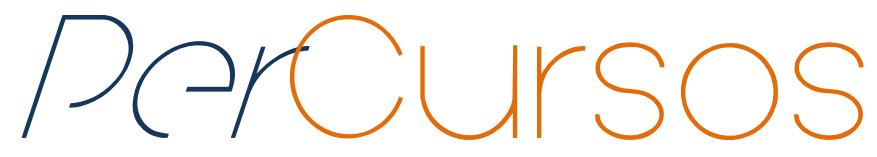

\title{
Interfases territoriales y construcción histórico ambiental del hábitat: tramo urbano Rio Otún, Pereira-Colombia
}

\begin{abstract}
Resumen
El artículo tiene como propósito la generación de una propuesta de construcción colectiva del hábitat rur-urbano en el subtramo 1 de la Operación Urbana Integral del tramo urbano del río Otún, desde el enfoque de interfases ambientales y mejoramiento de barrios para la contribución de la proyección ambiental del territorio. De este modo, se realizó una pequeña reconstrucción histórico-ambiental, yendo desde una formación a nivel de ciudad y asentamientos, para terminar en el área de estudio; posteriormente se destaca la importancia eco-sistémica de las cuencas Otún y Consotá; y se diagnostica el territorio teniendo como eje principal la planificación y gestión del río Otún desde una mirada de gobernanza construida a través de imaginarios colectivos, la cual posibilitó centrar y priorizar principios y estrategias para la construcción colectiva del hábitat. El trabajo se basó en la teoría de la Proyectación Ambiental y por ende, la inclusión de la dimensión ambiental en el proceso de planificación y gestión urbana, permitiendo pasar del diagnóstico del deterioro de las condiciones del hábitat, hacia la construcción social de una racionalidad ambiental. Se pueden establecer como soporte teórico principal, el enfoque de patrones e interfases ambientales a través de la caracterización de las relaciones actuales rururbanas y el análisis de la dinámica ecosistema-cultura como corriente alternativa de pensamiento ambiental complejo; es en este punto que la ciudad representa la principal expresión tangible del sistema sociocultural, construido y transformado, y es allí donde las interfases ambientales representan las interrelaciones que se establecen en un territorio.
\end{abstract}

Palabras-clave: Interfases ambientales; Apropiación; Imaginarios; Planeación y territorio.
Luisa Fernanda Durán Montes

Administradora Ambiental de la Universidad Tecnológica de

Pereira - Colombia. dumonto94@gmail.com

César Andrés Alzate Hoyos

Administrador Ambiental de la Universidad Tecnológica de Pereira - Colombia. csalzate@gmail.com

\section{Samuel Darío Guzmán López}

Economista, Magíster en

Desarrollo Sustentable por la Universidad Nacional de Lanus; profesor titular e investigador asociado de la Facultad de

Ciencias Ambientales -

Universidad Tecnológica de

Pereira-Colombia. sguzman@utp.edu.co

\section{Para citar este artículo:}

DURÁN, Luisa Fernanda Montes; ALZATE, César Andrés Hoyos; GUZMÁN, Samuel Darío López. Interfases territoriales y construcción histórico ambiental del hábitat: tramo urbano Rio Otún, Pereira-Colombia.

Revista PerCursos. Florianópolis, v. 16, n.30, p. 07 - 33. jan./abr. 2015. 


\section{Territorial interfaces and historical environmental building of habitat: urban stretch River Otún, Pereira- Colombia}

\begin{abstract}
This article has the purpose to generate a proposal for collective construction of rural-urban habitat in subsection 1 of the Integral Urban Operation urban stretch of river Otún, from the viewpoint of environmental interfacses and neighborhood improvement for the contribution of Projection Environmental Planning. For the development of this small historical environmental reconstruction was performed, ranging from a formation to city level and settlements, ending in the study area; further highlights the importance of the ecosystem Consota and Otún basins; and territory are diagnosed with the main axis of the planning and management Otún river from a look of governance built through collective imagination, which enabled focus and prioritize principles and strategies for collective construction of habitat. This work was supported on the theory of Projection Environmental Planning and thus the inclusion of the environmental dimension in the process of urban planning and management, allowing to move from diagnosis of deteriorating habitat conditions, to the social construction of rationality environmental. Can be established as the main theoretical support, approach patterns and environmental interfacses through the characterization of the present rural-urban relations and analysis of dynamic ecosystemculture as alternating current complex environmental thinking. At this point the city is the main tangible expression of the sociocultural system, built and transformed, and that is where environmental interfacses represent the relationships established in a territory.
\end{abstract}

Keywords:: Environmental interfacses; Empowerment,; Imaginary; Planning and territory.

\section{Interfaces territoriais e construção histórica ambiental do habitat: trecho urbano Rio Otún, Pereira-Colômbia}

\section{Resumo}

$\mathrm{O}$ artigo tem o intuito de gerar uma proposta de construção coletiva do habitat rur-urbano no subtrecho 1 da Operación Urbana Integral do trecho urbano do río Otún, desde o enfoque de interfaces ambientais e melhoramento de bairros para a contribuição da projeção ambiental do território. Deste modo, realizou-se uma breve reconstrução histórico-ambiental, partindo da formação em nível de cidade e assentamentos para terminar na área de estudo. Seguidamente, ressalta-se a importância ecossistêmica das bacias hidrográficas Otún e Consotá e, diagnostica-se o território tendo como eixo principal o planejamento e gestão do rio Otún desde um olhar de governança construída através dos imaginários coletivos, na qual possibilitou centrar e priorizar princípios e estratégias para a construção coletiva do habitat. A pesquisa se baseou na teoria da Proyectación Ambiental, assim como a inclusão da dimensão ambiental no processo de planejamento e gestão urbana, permitindo passar do diagnóstico do deterioro das condições do habitat até a construção social de uma racionalidade ambiental. Pode-se estabelecer como suporte teórico principal, o enfoque de padrões e interfaces ambientais a partir da caracterização das relações atuais rururbanas e a análise da dinâmica ecossistemacultura como corrente alternativa do pensamento ambiental complexo, é neste ponto que a cidade representa a principal expressão tangível do sistema sociocultural, construído e transformado e, é ali, onde as interfaces ambientais representam as interrelações que se estabelecem num território.

Palavras-chave: Interfaces ambientais; Apropriação; Imaginários; Planejamento e território. 


\section{Introducción}

Pereira, una ciudad de tamaño poblacional medio en el contexto colombiano (500.000 - 1.000.000 habitantes), capital del departamento de Risaralda e integrante del Área Metropolitana Centro Occidente-AMCO-, tiene un escenario de planificación denominado Operaciones Urbanas Integrales, donde una de ellas, se localiza en el Tramo Urbano del río Otún (OUI), en la que se destaca el sub-tramo 1 del río, donde el paisaje natural es inmediato y el ámbito visual es de amplia cobertura; además se encuentra en construcción el Parque Lineal que se puede definir como un intento de nuevo esquema urbano rural que busca la generación de espacio público que permita la resignificación y apropiación cultural del río Otún.

En este sentido, el ambiente se concibe como el resultado de las interacciones que se generan entre el sistema natural y el sistema cultural con sus implicaciones sociales.

La ciudad representa la principal expresión tangible del sistema sociocultural, construido y transformado continuamente por el hombre; es allí donde las interfases ambientales representan las interrelaciones que se establecen en un espacio territorial, en el cual se dan procesos de apropiación y transformación (PESCl, 2007, p. 58).

Por ende:

La inclusión de la dimensión ambiental en el proceso de planificación y gestión urbana permite pasar del diagnóstico del deterioro de las condiciones del hábitat, hacia la construcción social de una racionalidad ambiental, capaz de reorientar la evolución cultural del hombre en armonía con las condiciones y potenciales ecológicos del planeta (LEFF, 2000, p. 87).

En un contexto regional, las Áreas Metropolitanas con el fin de mejorar integralmente zonas de interés prioritario, diseñan Proyectos Urbanos Integrales (PUI en la ciudad de Medellín-Colombia), que en el caso de Pereira son denominadas Operaciones Urbanas Integrales (OUI); su principio y función es el mismo que se encuentra postulado en la Ley 388 de 1997, es decir, que los municipios a través de los Planes de Ordenamiento 
Territorial -POTs- tienen un instrumento que les permite planificar su desarrollo bajo criterios de sostenibilidad ambiental, humana, económica y de equidad social que respondan generalmente a los hechos metropolitanos identificados.

La investigación “Interfases Ambientales para el Mejoramiento de Barrios y la Construcción de Hábitat Colectivo. Caso de estudio: Sub-tramo 1 de la Operación Urbana Integral del Tramo Urbano del Río Otún, Conurbación Pereira-Dosquebradas", generó una propuesta de construcción colectiva del hábitat rur-urbano en el sub-tramo 1 de la OUI del tramo urbano del río Otún, desde el enfoque de interfases ambientales y mejoramiento de barrios a través de la caracterización de las relaciones actuales de las interfases rururbanas y el análisis de la dinámica ecosistema-cultura; finalmente se proyectó ambientalmente la construcción del hábitat colectivo en el sub-tramo 1. La metodología base escogida para realizar el proyecto fue la "Proyectación Ambiental” propuesta por el Foro Latinoamericano de Ciencias Ambientales (FLACAM). No obstante, en el desarrollo del proyecto se utilizaron técnicas y herramientas propias de otras metodologías y métodos que permitieron analizar conflictos y potencialidades a nivel territorial.

\section{Formación de la ciudad de Pereira y cronología de asentamientos}

El origen de Pereira, está en el Cartago viejo que fundó el conquistador español Jorge Robledo en 1536, lugar donde los Quimbayas ejercían dominio sobre el territorio que tenía el nombre de Consotá y eran liderados por un cacique que llevaba el mismo nombre. Los enfrentamientos con los indígenas obligaron a sus fundadores a trasladar Cartago al lugar que ocupa hoy, y en 1863 la convergencia de arrierías que transitaban por estas tierras y cuando se habían extinguido los Quimbayas, llevó a un grupo de personas a asentarse en el sitio de la Pereira actual. Eran tierras vírgenes y prósperas en su mayoría, que ofrecían a sus nuevos pobladores muchas oportunidades para habitarlas.

Se trataba de un territorio geocéntrico de poblaciones anteriores como Manizales, Cali, Riosucio, Calarcá y la misma Cartago, rodeado de selvas y bosques primarios, entre los que se destacaban ecosistemas importantes como Tatamá y Los Nevados. En este último, nacen los ríos Otún y Consotá, que forman en sus cuencas medias, la superficie 
que acogió la aldea que Ilamaron Pereira y donde su asentamiento inicial, definió la cuadrícula o damero español convencional con el que se erigieron las poblaciones de la época colonial.

Los ríos Otún y Consotá que llevan nombres Quimbayas, fueron la periferia de la Pereira de fines del siglo XIX y luego durante el siglo XX, la expresión física de la marginalidad social de la ciudad que emergió como urbe comercial e industrial durante la primera mitad del mismo y luego hasta los albores del nuevo siglo, en el límite sanitario o depósito de los residuos generados por la metrópoli y escenario de los conflictos ambientales más relevantes de la nueva capital.

Los bordes norte y sur de la ciudad, se convirtieron en el espacio que configuró su subnormalidad habitacional y en escenario para una planificación convencional que no consultó dichos hechos como una nueva realidad y que los llevó a convertirse en la expresión de una centralidad urbanística que no tuvo en cuenta la totalidad del territorio (GONZÁLEZ, 2011, p. 115).

Yendo un poco más atrás en la historia, se encuentra que:

El poblamiento de Pereira data de hace 10.000 años A.P. Es posible denotar que los asentamientos humanos han tenido un patrón que guarda relación con el aprovechamiento de suelos fértiles (constituidos por cenizas de los volcanes del Ruiz, Santa Isabel, Tolima y Quindío) y de recursos bióticos y minerales, evidenciándose en el sistema de enterramiento, expresión original de sus costumbres y creencias funerarias (GONZÁLEZ, 2011, p. 33).

El territorio de la Provincia Quimbaya o Cauca Medio, corresponde en parte al área de lo que en la actualidad se denomina Eco Región Eje Cafetero (GONZÁLEZ, 2011); con características geográficas que comenzaron a definir las dinámicas de habitabilidad frente al territorio. Un ejemplo de ello, es que: 
El Consotá corre más alto que el Otún. El Cañón del Otún es más profundo que el del Consotá, corre por un tramo más estrecho y el Consotá, que genera un valle, es mucho más apto para la vida humana, generando playas y llanuras que debieron ser ocupadas en el pasado. En principio la evidencia muestra que desde el 10.000 hasta el 5.000 A.P. sus habitantes fueron cazadores-recolectores y desde el año 5.000-3.000 A.P. aproximadamente, fueron horticultores (LÓPEZ, 2008, p. 34).

Con relación a ello, López Castaño expone que la ocupación de la cuenca del Consotá ha sido más pronunciada que la del Otún, y poblacionalmente, la ocupación del Otún es tardía con relación al Consotá, ello debido a los usos del suelo para la agricultura (LÓPEZ, 2008).

En términos generales se resalta que en la zona plana del valle del río Cauca, de acuerdo con Julio César Cubillos (1948), se pueden clasificar tres modalidades de asentamientos prehispánicos: poblamiento nuclear simple, formado por caseríos; poblamiento lineal, a lo largo de las corrientes de agua; y poblamiento disperso, sobre terrazas artificiales.

De este modo, es importante establecer los diferentes patrones de ocupación ya brevemente descritos con los hechos históricos emergentes causales de la dinámica particular de la región. Tres grandes momentos se identifican desde estos remotos antecedentes hasta comienzos del siglo XX, caracterizados sucesivamente por el predominio indígena, español y antioqueño-caucano, produciéndose en este último varias oleadas. Poblamiento indígena: migrantes de origen principalmente centroamericano que iniciaron la presencia humana en la región. Sus descendientes, a mediados del siglo XVI, desarrollaron una cultura cumbre, la Quimbaya, que a la llegada de los hispanos ya había presenciado incursiones de los indios Caribe (GONZÁLEZ, 2011, p. 33).

Al respecto, la población aborigen fue prácticamente exterminada por los hispanos en menos de 100 años. Teniendo en cuenta la importancia de las culturas indígenas, un hecho como tal, cambió las relaciones que posteriormente irían a determinar la forma de interacción particular de los colonos con los habitantes que consolidarían este territorio, precisando en este caso que el despoblamiento particular de 
la época por parte de los indígenas y los afro-descendientes demarcarían la senda del ulterior individualismo y fuerza antioqueña en el desarrollo social y económico de Pereira. En este sentido, quizás un factor determinante es la colonización antioqueña y caucana, que ocurre entre 1780 y 1900 aproximadamente. En todo caso, la característica de "territorio de paso" inicialmente, y de encuentro posterior, generó interacciones económicas, sociales y políticas que antecedieron al origen de Pereira y que la proyectaron como ciudad cosmopolita.

La colonización antioqueña tuvo tres momentos: uno de tipo espontáneo con avances de colonos pobres (principalmente arrieros) entre 1785 y 1810; el siguiente entre 1820 y 1860 de más sentido gremial en un movimiento que logró incorporar capital económico al proceso, colocando al territorio como uno de los de mayor dinámica poblacional (DUQUE; FRIEDE; JARAMILLO, 1963). Y un tercer momento "a partir de 1870 que generó hechos como la fundación de la ciudad a partir de la colonización, que organizaron exploradores agropecuarios de gran envergadura" (GONZÁLEZ, 2011, p. 49).

Desde el punto de vista urbanístico, la década que va de 1870 a 1880 fue un periodo en el que la nueva villa (Pereira) creció aceleradamente. Como resalta Duque (1963), el 20 de Enero de 1870 es erigida en distrito con derecho a tener cabildo y, a partir de este año recibe una nueva ola de inmigrantes procedentes sobre todo de Antioquia. En este mismo año se hace un nuevo trazado de sus calles y plazas y se levanta un plano de la ciudad, obra que ejecuta el ciudadano inglés Guillermo Flecher.

El desarrollo de Pereira tiene muchas instancias históricas, pero se ha de resaltar en este apartado el periodo constituido entre 1905 y 1930 donde pasa a convertirse en una de las diez ciudades más importantes del país. Para la década de los 40 los procesos de poblamiento se desarrollaban bajo el esquema concéntrico a las plazas principales manteniendo su forma alargada en el sentido Este-Oeste; sin embargo, la década de los 60 fue bastante compleja por la alta concentración de personas venidas de otras regiones del país en busca de hospitalidad y amenazadas por la violencia política (ROJAS, 2003, p. 156).

Antes de la década del 50 el río Consotá era una barrera natural, un límite físico y topográfico. Pero el crecimiento hacia el sur se dio con la 
consolidación de nuevos barrios, siendo Cuba el primero de ellos. Desde entonces, el casco urbano empezó a ocupar las márgenes del río Consotá en condiciones inadecuadas y de alto riesgo por las características de informalidad de los nuevos asentamientos; a mediados de los años 90 se inició el proceso de planificación que concluiría con la promulgación del acuerdo 18 del 2000, que dio origen al Plan de Ordenamiento Territorial, POT, en donde se evidencian las dinámicas expansivas de Pereira y se proponen soluciones para amortiguar dichos impactos urbanísticos (GONZÁLEZ, 2011, p. 47)

\section{Área de estudio}

Después de un breve recuento cronológico a un nivel más general de los asentamientos formados en la región, se presenta a continuación un análisis más focalizado para el área de estudio que da luces a lo que sería una construcción históricoambiental del tramo urbano del río Otún y su formación cronológica a nivel barrial. En un primer acercamiento se mencionan algunos hitos que fueron representativos para el desarrollo de la comuna Oriente, sector donde está ubicada la mayor parte del área de estudio. Esta breve recopilación histórica se basó principalmente en el estudio del desarrollo físico espacial y social del municipio de Pereira realizado por John Alberto Vanegas (1997):

En 1924 se construye el puente férreo sobre el río Otún, denominado "Puente Mosquera", que le daría continuidad al tramo del ferrocarril de occidente que desde Buenaventura (Departamento del Valle) uniría al departamento de Caldas con el puerto marítimo sobre el Pacífico colombiano, permitiendo el transporte de la producción para exportación de la región, sobre todo del café, que ya se avizoraba como el producto más importante del país y que tenía en esta zona, una de las áreas de mayor producción.

En el año 1942 el municipio construye el Estadio Alberto Mora Mora, en el sector de Libaré, escenario deportivo emblemático de la ciudad el cual le dio reconocimiento regional y nacional.

Para 1952 se establece en el sector la fábrica de Hilos Cadena S.A y para 1957 se termina el montaje de su línea completa de fabricación, (ASOCIACIÓN NACIONAL DE 
INDUSTRIALES, 2001). En 1992 cambió su razón social a Coats Cadena S.A., esta empresa contribuyó a la economía del país y especialmente de la región con la creación de gran cantidad de empleos directos e indirectos (GIL; ALVAREZ, 2011).

La urbanización del sector se desarrolló a partir de la década del 50, inicialmente con asentamientos informales, con la conformación de los barrios Ormaza, Santander y San Francisco que se extendieron hacia el sector oriental del río Otún (ROJAS, 2003).

Cabe resaltar que la información presentada a continuación pertenece a un tipo de cronología recolectada en su gran mayoría de fuentes primarias; moradores que aún mantienen un relato oral de gran valor histórico. La antigua vereda Libaré que anteriormente pertenecía a la vereda Gaitán, se funda en 1955 siendo en un principio un caserío, zona donde se encontraban y se reunían los arrieros provenientes de varios municipios, caracterizó la conexión entre la zona rural y urbana del municipio.

Para el año de 1960 nace el barrio Chicó Restrepo, que se construyó a partir de un plan de vivienda, una parte por autoconstrucción y otra construida por la fundación San Vicente de Paul para mujeres viudas ${ }^{1}$. Dos años después se funda en una primera etapa el barrio Kennedy (1962), lo que se da en el marco de la ejecución del programa de ayudas del gobierno de Estados Unidos, conocido como la Alianza para el Progreso, a través del INSCREDIAL (Instituto de Crédito Territorial). El otro sector (1968) se construye a través de un Plan de Unidades Básicas de Vivienda para los empleados de la empresa Hilos Cadena. Estas viviendas de tipo popular se hicieron por autoconstrucción, para entonces sólo entregaron lotes con servicios y el instituto otorgó préstamos para culminarlas.

Antes de que se construyera la segunda parte del barrio Kennedy, para el año de 1964 y mediante un Plan de vivienda, se funda el barrio Simón Bolívar, que comenzó como un proceso de autoconstrucción por medio de invitaciones, el barrio comenzó solo con 50 casas, y contó con el apoyo del Servicio Nacional de Aprendizaje -SENA-.

Para finalizar la década de los 60 en esta breve cronología barrial, en 1965 es construido por empleados de Empresas Públicas de Pereira, el barrio El Pízamo. Cabe agregar que, los barrios Simón Bolívar, Chicó Restrepo y La Rivera fueron desarrollados

\footnotetext{
${ }^{1}$ Bernardo Gálvez, Presidente de la Junta de Acción Comunal del barrio Chicó Restrepo (JAC), Pereira 2011.
} 
por planes de vivienda, promovidos por particulares y respondían a diversos intereses políticos y económicos. Así mismo, se denota que la mayoría de barrios que nacieron en la década de los 70 son producto de gestiones políticas, que conllevaron a la constitución de barrios relativamente muy pequeños de una o dos manzanas (CARDER-UCPR, 2008).

Para el año 1974 nace en un predio cafetero, el barrio La Rivera. Construido a través de invitaciones, se fueron formando las viviendas, luego se buscó la personería jurídica y cada casa fue recibiendo su respectiva escritura. La construcción total del barrio se hizo a través de un plan de vivienda, para poder lotear el terreno².

En el año de 1978 se funda el barrio San Gregorio; con la donación de lotes de una fundación, este sector fue construido de manera conjunta, en ese tiempo ${ }^{3}$.

Esta década finaliza con la formación del barrio César Nader (1979). Lo que inicialmente era una laguna y una pesebrera, se va habitando, primero el Nader Nader por personas que venían del barrio Hernando Vélez Marulanda, comenzó con 49 viviendas. Con ayuda de representantes políticos como el Dr. César Nader, lograron llegar a un acuerdo con la Alcaldía de turno y el dueño del predio para pagar los lotes.

El barrio está divido en dos secciones: Nader Nader y La Playa-Libaré, anteriormente esta última sección pertenecía al barrio Chicó Restrepo, pero al momento de conformar la JAC se decidió entregarla al Nader Nader por la vecindad de los mismos 4 .

La última gran referencia se da en el barrio Altos del Otún en 1998. El terreno es un predio del señor Guillermo León Valencia, que con un proyecto de interés social se ha venido construyendo desde hace 13 años. Algunos propietarios han sido beneficiados por la ayuda de la Iglesia Luterana de Colombia 5 .

En cuanto a las transformaciones y/o adecuaciones del terreno, si bien en la llanura de inundación del río, que es donde se consolida la mayor parte de la comuna, no se realizaron mayores alteraciones a la fisonomía. Las laderas adyacentes sí fueron producto de intervención para la

\footnotetext{
${ }^{2}$ María. Quiceno, Presidenta de la Junta de Acción Comunal del barrio La Rivera (JAC), Pereira 2011.

${ }^{3}$ Tarcisio. Barreto, Presidente de la Junta de Acción Comunal del barrio San Gregorio (JAC), Pereira 2011.

${ }^{4}$ Hernán. Osorio Presidente de la Junta de Acción Comunal del barrio César Nader (JAC), Pereira 2011.

${ }^{5}$ Consuelo Torres, Presidente de la Junta de Acción Comunal del barrio Altos del Otún (JAC), Pereira 2011.
} 
localización de asentamientos como Chicó Restrepo, Simón Bolívar, San Gregorio y El Pizamo (ROJAS, 2003, p. 167).

\section{Importancia ecosistémica de las cuencas Otún y Consotá}

Como se explicó en los párrafos anteriores, la investigación buscó conocer las interfases territoriales del tramo urbano del rio otún a través de una construcción histórico-ambiental del hábitat colectivo, dicha construcción se soportó sobre los postulados del pensamiento ambiental desarrollado en Latinoamérica principalmente por Augusto Ángel Maya en Colombia y Enrrique Leff en México, a partir del pensamiento emergente del Club de Roma (primera conferencia mundial en 1968 y publicando Los Límites al Crecimiento en 1972), donde se permitió en el ámbito de la reflexión académica, como apunta Ana Patricia Noguera (2009) que el pensamiento emergente del Club de Roma fuese emergiendo lenta, dolorosa y progresivamente, gracias a estos dos pensadores, que se abrieran caminos diferentes e invitaron a abrir nuevos caminos en clave de un pensamiento latinoamericano (NOGUERA, 2009).

De esta manera se presenta la importancia ecosistémica de las cuencas Otún y Consotá para posteriormente entrelazarlas en un ámbito de gestión y planificación.

En primera instancia se encuentra la cuenca hidrográfica del río Otún la cual nace en el caño Alsacia, afluente de la Laguna del Otún, a una altura de 3.980 m.s.n.m. Tiene una longitud total de $67 \mathrm{~km}$, siguiendo una trayectoria en sentido este-oeste hasta confluir en el río Cauca a los 875 m.s.n.m. En su recorrido cruza la conurbación PereiraDosquebradas en sentido oriente-occidente, siendo a su vez el límite entre estos municipios (DURÁN, 2012) De este mismo modo, el Consotá nace en la vereda el Manzano a 2.150 m.s.n.m, pasa por el costado sur del tramo urbano de Pereira, atravesando la ciudad de oriente a occidente, para desembocar en el río la Vieja a una altura de 930 m.s.n.m. entre Cerritos y Cartago (CARDER, 2007).

La cuenca del río Otún al igual que la del Consotá hacen parte de la Ecorregión del Eje Cafetero perteneciendo al corredor Parque de los Nevados y al Parque Regional Natural Barbas-Bremen respectivamente. 
Si bien ambas cuencas cumplen un rol determinante en la regulación ecosistémica, el río Otún ha sido visto como la gran reserva hídrica de la región, mostrando un gran potencial ecosistémico principalmente por su oferta paisajística y de biodiversidad para la localidad, que permite además un aprovechamiento múltiple como el consumo de agua humano y de uso doméstico, para actividades agrícolas y pecuarias, generación eléctrica, receptora y diluyente de aguas residuales, recreación y preservación de flora y fauna. (DURÁN, 2012, p. 43)

Por otro lado la cuenca del río Consotá presenta suelos y climas aptos para la agricultura; sin embargo, en la cuenca media y baja prevalecen los usos residencial, industrial y ganadero (UCPR, 2008), lo que provoca gran contaminación del río y sus afluentes por vertimientos del orden del $67 \%$ de las aguas residuales de la ciudad de Pereira, siendo críticos los niveles de calidad principalmente en las estaciones de Boston, El Oso y El Tigre.

Sobre todo, entre la década de los 50 - 80 se evidencia un proceso de consolidación de aproximadamente un $20 \%$ de los asentamientos a orillas del río Consotá, como son Rocío Bajo, Rocío Alto y el barrio Providencia, a tan solo 300 metros del río Consotá, así como la zona de Cuba y posteriormente los asentamientos de Boston y El Poblado, (DIAZ, 2007, p. 69)

Teniendo en cuenta el delicado estado ambiental de ambas cuencas, estas siguen cumpliendo funciones de carácter social, ecológico, de preservación, de producción de oxígeno, y polo de atracción de la región. Solo el río Otún es la fuente utilizada como abastecedora de agua para el consumo de los habitantes de Pereira y gran parte de los de Dosquebradas (los cuales representan el $63 \%$ de la población del departamento de Risaralda) (DURÁN, 2012). 


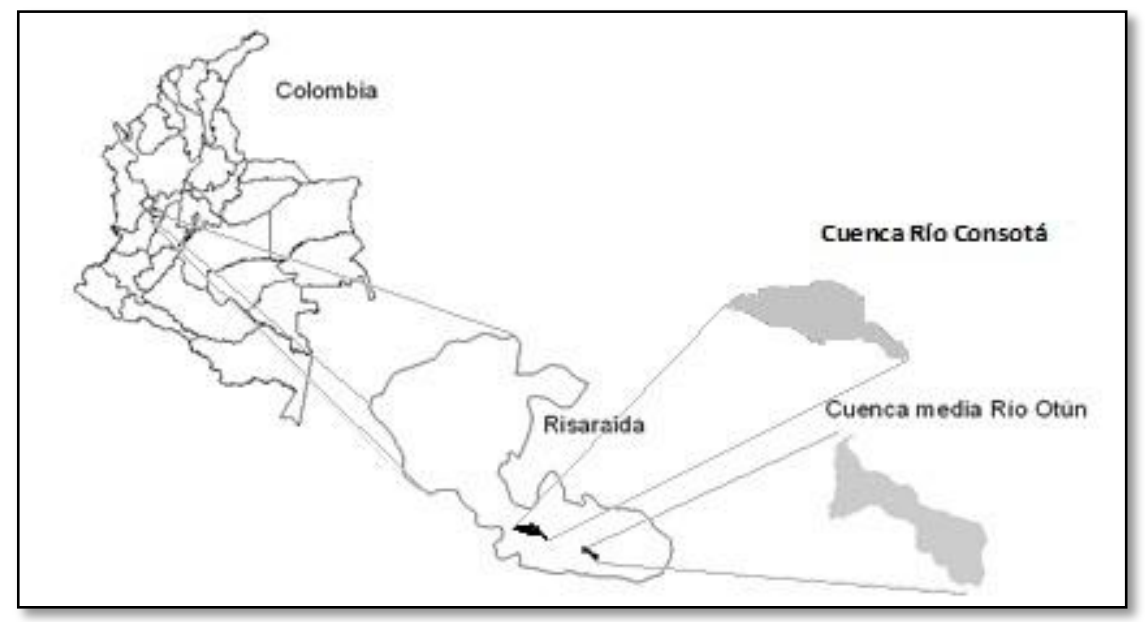

Mapa 1 - Georeferencia de la cuenca media río Otún Fuente: CARDER, 2008.

Modificaciones hechas por los autores

\section{Metodología del proyecto}

La metodología base escogida para la realización del proyecto fue la "Proyectación Ambiental" propuesta por el Foro Latinoamericano de Ciencias Ambientales (FLACAM). Como explica Pesci (2000, p. 34):

El proyecto ambiental puede tener énfasis en distintas disciplinas o según el conflicto de que se trate, pero la lista de componentes de un proyecto ambiental, no necesariamente va en orden sucesivo, ni tampoco se deben considerar todos los pasos indispensablemente En el desarrollo del proyecto se utilizaron técnicas y herramientas propias de otras metodologías que permitieron analizar conflictos y potencialidades a nivel territorial.

Dentro del proceso metodológico propuesto en FLACAM se aplicaron dos métodos holísticos: percepción e interfases.

El primero consiste en un acercamiento empírico en el trabajo de campo, utilizando esencialmente los sentidos para captar las relaciones que se establecen en el ambiente; y el segundo, consiste en concebir las interacciones entre dos o más ecosistemas como el área de mayor intensidad de intercambios de flujos de información, 
materia y energía, registrando los fenómenos críticos de cada ecosistema en relación con el contexto.

Con este enfoque se abordó el reconocimiento de los fenómenos de causa-efecto del territorio, como también de sus verdaderas redes y sistemas de alta complejidad; por ende, la metodología busca conocer las realidades que nacen de la lectura que cada actor le da al territorio. Mejorar el estado de una situación problemática a una de mayor cualidad, o de mayor deseabilidad para la comunidad, es el propósito de la "Proyectación Ambiental"

Por lo anterior, se plantea una visión holística de esta problemática de acuerdo a las características del territorio y a los capitales (humanos, económicos, natural, entre otros) que en este intervienen. Precisamente por eso, se escogió y se hizo énfasis en la Interfase como método de estudio para el análisis de los sistemas y sus interrelaciones. Según Pesci (2007), la teoría de interfases ambientales se elaboró para comprender y actuar eficazmente sobre los sistemas complejos y su alta e impredecible dinámica de cambio.

Para el desarrollo de los objetivos específicos Se hizo una revisión documental, precedida de un proceso de recolección y análisis de la información para la consolidación de las percepciones e imaginarios de la comunidad. Después se llevó a cabo un proceso de análisis del diagnóstico que consistió en una serie de procedimientos tales como descripción de contextos culturales, ambientales, políticos, históricos, configuración integrada de percepciones e imaginarios, entre otros.

Finalmente, se desarrolló a través de una consulta a expertos una propuesta enfocada en la construcción colectiva del hábitat, evaluando los procesos generadores de conflictos y potencialidades que se consolidaron en principios, lineamientos, estrategias y proyectos.

\section{Diagnóstico del territorio}

Es de suma importancia aclarar que, en este caso de estudio las interfases ambientales serán entendidas como las interrelaciones socio- 
culturales que se establecen en un espacio territorial, en el cual se dan procesos de apropiación y transformación, debido a que el concepto de interfases presenta una noción de gran valor analítico y operativo en el abordaje de las áreas urbanas y territoriales complejas (PESCI, 2007, p. 76).

En pocas palabras, las ciudades son un ámbito experimental muy apropiado para aplicar el concepto de interfases, pues se trata de un sistema ambiental (ecosistema y cultura) muy complejo e interactivo (PESCl, 2007).

En este trabajo se reconocen dos interfases presentes en el sub-tramo 1 de la Operación Urbana Integral (OUI) del tramo urbano del río Otún (TURO): el punto de máxima conflictividad en la dialéctica campo-ciudad y el punto de máxima interacción social entre grupos diferentes.

Una de estas interfases se encuentra entre ecosistemas predominantemente naturales y un ecosistema predominantemente artificial urbano, donde el conflicto de intercambios logrados (o impedidos) es de tipo esencialmente económico. Es decir, que no se trata sólo de evitar los males manifiestos de la interfase peri-urbana, sino también recuperar sus virtudes ocultas (PESCI, 2007).

Cuando en el territorio se reconoce y acepta la presencia de la cuenca dentro de la planificación territorial, inmediatamente se facilita la relación entre sus habitantes y de estos con el ecosistema, independientemente de si estos se agrupan dentro de dicho territorio en comunas delimitadas por razones político-administrativas, debido a su dependencia común a un sistema hídrico compartido, a los caminos y vías de acceso (DOUROJEANNI, 1994, p. 10).

Es necesario, entender que la cuenca es además una unidad natural que sirve de eje estructurante de la planificación y gestión territorial, articulando estos procesos a un propósito mayor que sería el desarrollo sustentable; las cuencas hidrográficas permiten identificar el efecto negativo de las acciones que el hombre realiza sobre su entorno, sobre todo porque se refleja en la contaminación del agua; siendo este recurso eje de 
articulación para coordinar las acciones de crecimiento económico y equidad (DOUROJEANNI, 1994).

Se reconoce que la cuenca del río Otún es proveedora de bienes y servicios ambientales, tales como: madera, pesca, agua potable, fuente de energía y ecoturismo, entre otros. De esta forma, el río constituye el principal patrimonio natural e histórico que determinará el futuro socioeconómico del territorio.

Sin embargo, la facilidad de acceso al río por su conexión a los centros urbanos viene motivando el desarrollo de actividades que lo amenazan y a los recursos naturales asociados. Así, la ubicación estratégica del río Otún constituye una gran oportunidad y, paradójicamente, una inmensa debilidad que exige adoptar decisiones y emprender acciones entre distintos estamentos de la sociedad.

En dicho tramo ubicado dentro del área urbana del municipio de Pereira y el área rural de los municipios de Dosquebradas y Santa Rosa de Cabal, los habitantes viven en su cotidianidad las consecuencias de la contaminación del río, esta se da en zonas de entrega de aguas servidas industriales, básicamente de barrios aledaños que carecen de tratamiento.

Por otra parte, la riqueza escénica de la cuenca próxima a la zona urbana, favorece el acceso de visitantes que buscan aprovechar los bienes y servicios ambientales existentes, al tiempo que los pobladores locales ven en la prestación de servicios turísticos una alternativa de ingreso económico. Las actividades turísticas y recreativas no planificadas carentes de una adecuada infraestructura sanitaria, y la frecuente alteración en el cambio del uso del suelo, generan una serie de conflictos ambientales en la margen del río.

En pocas palabras, el río como línea de interacción entre la conurbación PereiraDosquebradas, se convierte en un factor importante y partícipe del desarrollo de ambas ciudades. La cuenca cumple funciones de carácter social, ecológico, de preservación, de producción de oxígeno, diversión y polo de atracción para la ciudad; sin embargo, la contaminación lo convierte en un lugar de alta sensibilidad ambiental. 


\section{Conurbación Pereira-Dosquebradas, AMCO y el subtramo (OUI)}

Conurbación o conurbano se refiere al proceso de crecimiento, expansión y encuentro de dos ciudades. Las conurbaciones se destacan más por su capacidad de diferenciar las otras ciudades que la conforman, que por su posible continuidad física, además se encuentran unidas por intereses comunes. Su estructura urbana permite las interrelaciones entre los diversos núcleos, debido a que existe una red de comunicaciones y sistemas de transporte que posibilitan sus intensas relaciones (GIRALDO, 2007, p. 28).

En el área conurbada acontecen tres hechos trascendentales: el crecimiento demográfico, el uso del suelo y el corredor urbano regional como tipología de articulación por el acceso al transporte, a la provisión de agua y por la localización de la industria (ISAZA, 2008).

La conurbación como concepto geográfico, hace referencia al proceso y resultado del crecimiento de varias ciudades o centros urbanos que resultan integrándose en un solo sistema, pero siempre manteniendo la relativa independencia funcional y dinámica (ISAZA, 2008).

Una propuesta de gestión ambiental para la Subregión 1, tiene como característica central el énfasis en la problemática ambiental urbana: contaminación de ríos y quebradas por inadecuado manejo de residuos líquidos y sólidos, el no control de emisiones de fuentes fijas y móviles, entre otros (CARDER, 2007)

Al respecto, el Área Metropolitana Centro Occidente ha formulado diversos macroproyectos Ilamados Operaciones Urbanas Integrales, que responden a los hechos metropolitanos. Para efectos del caso de estudio se hace referencia a la Operación Urbana Integral del Tramo Urbano del río Otún en el sub-tramo 1.

La OUI se orienta a mejorar el espacio público, utilizando terrenos vacíos pertenecientes a instituciones públicas como el AMCO y la CARDER, dado que dicha zona se encuentra ubicada en una interfase urbano-rural, la mayor parte del suelo se considera 
según el POT como suelo de conservación y protección y la infraestructura presente en los barrios no es suficiente, al mismo tiempo de estar en regular estado.

En resumen, el tramo urbano del río Otún es un área de especial importancia ecosistémica, esta área será tratada como un macroproyecto teniendo en cuenta la importancia que tiene la ladera norte y el tramo urbano del río Otún como hechos metropolitanos para los municipios de Pereira y Dosquebradas, dentro de la cual se prevé la ejecución del proyecto Malecón Otún.

\section{Relaciones territoriales del subtramo (OUI)}

El territorio como constructor socio cultural, representa el carácter identitario en un espacio de interacción socio ambiental. En él, las percepciones, imaginarios y cosmovisiones se conjugan para manifestar un entorno.

El tramo urbano de la cuenca media del río Otún, tiene en su origen, que se materializa cuando se habita dicho territorio, una impronta rural, de camino de paso, de fonda de arrieros, de trueques de mercancías y de deseos de pernoctar por fin y quedarse para siempre en el lugar que les ofrece el descanso, o la sensación de sentimiento del deber cumplido.

Esta investigación arrojó que el porcentaje más alto de sus habitantes actuales ${ }^{6}$, el $64 \%$, coinciden en que su territorio representa vida, como la manifestación de tenerlo y encontrarlo todo, complementándose con un 13\% que manifiesta que la representación preponderante es su biodiversidad y un $11 \%$ que siente al río como articulador no solo natural, sino también al entorno social.

Las palabras más representativas para los habitantes cuando piensan en su barrio, son seguridad, bienestar y unión, que reflejan la certeza, calidad de vida y auto reconocimiento frente a su entorno.

\footnotetext{
${ }^{6}$ Fueron entrevistados 9 líderes barriales y encuestadas 90 personas, esto de acuerdo con una muestra aleatoria simple aplicada a una población total de 1.364 habitantes del área de estudio, según información institucional del 2007. Del segmento poblacional encuestado, es posible caracterizarlo de la siguiente manera: $60 \%$ mujeres y $40 \%$ hombres, de los cuales el $27 \%$ de la población tenía entre 10 y 30 años; $67 \%$ entre 31 y 60 años; y $6 \%$ mayores de 60 años.
} 
La comunidad del sector dice sentirse bien en casa (29\%), un 19\% disfruta del paisaje natural y un 13\% prefiere el parque del barrio Kennedy como lugar de encuentro y espacio de relaciones. El arraigo social por su entorno residencial, refleja la tradición de las familias paisas, que reunidas alrededor de sus costumbres y prácticas sociales, tienen a dichos encuentros como una forma, quizás la mejor, de mantener a la familia unida.

Como necesidades más relevantes, los habitantes manifiestan (58\%), que la infraestructura (vías, saneamiento básico, espacio público) representa el déficit mayor de su entorno para la calidad de vida. Como asentamientos derivados de un camino de arrieros, los planificadores de la ciudad y los gestores sociales que desarrollaron los barrios del sector, poco concibieron la necesidad de espacios abiertos y dotados de infraestructura urbana necesaria para aquella zona de la ciudad que llegaría a demandar los mismos.

El 78\% sienten que no es necesario cambiar nada, en una percepción que tiene un fuerte arraigo por su entorno natural y la oferta paisajística que este les ofrece y que les significa conformidad por su hábitat.

Los destinos más frecuentes para los habitantes, son el parque Kennedy y el corregimiento La Florida, en una clara manifestación de los dos lugares entre los que se dibuja la vía principal de la zona, como eje articulador de su territorio y que pueden ser la clara manifestación de la necesidad de intervenciones urbanísticas que pudieran dar respuesta a otras formas de expresión espacial de la comunidad.

El atractivo turístico de La Florida, representa una oportunidad para el comercio formal e informal de la zona (tiendas, artesanías, alimentos, etc.), conscientes de que puede ser a la vez una amenaza, puesto que la capacidad de carga de la cuenca para atender la demanda ecoturistica puede llegar a niveles de saturación e impactos que afecten la oferta ecosistémica.

La Florida, es la mayor referencia del territorio nororiental de Pereira y con ello, se corre el riesgo de desconocer a los barrios allí asentados en el trayecto hacia los destinos turísticos de la ciudad y que pueden conllevar al olvido y desatención de necesidades de la que puede ser una zona amortiguadora. Son varios los proyectos de los que se hablan, 
impactarán positivamente el área, pero no son muchas las razones para creerlo, pues obras como el corredor ecoturistico Otún, siempre fue más un proyecto que una realidad y su estado de ejecución es prueba de ello. También se habla de un teleférico para conectar La Florida con Pereira, sin clara noción de cómo beneficiará al subtramo urbano.

En la que puede ser la mayor oportunidad de Libaré, está a la vez su mayor amenaza; la naturaleza referente a la cuenca y a la zona de amortiguación del Parque Nacional de los Nevados, puede ser la redención para la comunidad del sector, como lo proclaman quienes lo viven a diario, sus ciudadanos, que entienden a su vez que dicha riqueza natural no debería convertirse en su maldición.

\section{Propuesta de construcción de un hábitat colectivo}

Esta propuesta se enfocó en la re-significación del territorio tanto en su dimensión física como simbólica a partir de los lazos de pertenencia y arraigo, pero se ha evidenciado, no sólo en esta investigación sino también en otros proyectos que, la participación de los habitantes es deficiente y no está direccionada a la búsqueda de propósitos comunes; de este modo se pretende a través de lineamientos y estrategias de integración, fortalecer la identidad, los procesos de territorialización y de empoderamiento local.

Los principios, lineamientos, estrategias y proyectos representativos subsiguientes son elaborados con base en la consulta a expertos como resultado de la primera etapa del proceso metodológico, el cual además incluye recolección y análisis de diversos referentes teórico-empíricos. Al respecto, se identifican y se proponen unos principios y sub principios:

Participación entendida como un proceso: Las comunidades se reconocen como actores de su historia a nivel social, político, económico y cultural; por ende tienen el derecho y la responsabilidad de intervenir de manera consciente en la toma de decisiones sobre los asuntos que las afectan. 
a) Formación de actores sociales para que se conviertan en "autores de ciudadanía”.

b) Participación real de la ciudadanía y las comunidades locales (toma de decisiones y respeto por las autonomías locales con base en la identidad, la pertenencia y el empoderamiento de los habitantes).

Conservación de paisaje: Buen manejo de los recursos físicos, estéticos y funcionales relacionados directamente con la identidad de las comunidades; promueve paralelamente buen manejo de los bienes naturales y culturales, mediante patrones de producción y consumo compatibles con los límites de la sustentabilidad, forjándose un "Paisaje Identitario".

a) Interconexión entre ciudades y parajes del tramo del río Otún percibida no solo como parte de la oferta turística sino como constituyente de la identidad regional.

b) Superar la invisibilidad del territorio (lo que no se ve y no se siente), y pasar a su modelación integral, ética y estética, donde sea la ecoforma la que defina la prioridad en las decisiones territoriales y las interfases, el patrón esencial de modelación.

c) Pasar de la concepción fundamentalista de recursos naturales a preservar, a la modelación integrada del paisaje (paisaje diseñado agregando a los valores del sitio natural la "genialidad del lugar", es decir la interpretación cultural y los referentes multisignificativos de este).

d) Integración eficaz de las funciones de urbanidad y conservación del patrimonio cultural con un paisaje natural sustentable.

Integración urbano/rural: Evita las relaciones inconexas entre lo urbano y rural garantizando la evolución de las prácticas productivas y la ocupación del territorio, aproximando los beneficios de la ciudad al medio rural (conectividad, equipamientos, entre otros) y el soporte ambiental del medio rural hacia lo urbano.

a) Producción integradora de la ciudad: Establece alianzas de responsabilidad colectiva para la toma de decisiones de planeación. Introduce patrones integrales de 
construcción de ciudad. Garantiza la integración de saberes y ámbitos físico-espaciales entre las diversas instancias municipales y las instituciones de la comunidad.

\section{Lineamiento 1. Participación comunitaria en el proceso de gestión ambiental}

Gestión y empoderamiento de la comunidad: involucrar a la comunidad en la identificación de problemas y en el diseño de instrumentos de gestión, incorporando el conocimiento de quienes resultan ser los verdaderos "especialistas", ese conocimiento que sobre su medio poseen quienes protagonizan los problemas estudiados, y además quienes los "viven".

a) Proyecto representativo: Celebración de la fundación; consiste en el trabajo conjunto entre los barrios para festejar las fechas de fundación con un festival que conmemore su historia. La temática de dicho festival podría estar enfocada en las tradiciones.

Recualificación y fortalecimiento de los equipamientos con participación comunitaria: el municipio deberá destinar recursos a la actualización de los equipos existentes y su vinculación con cadenas productivas, tanto locales como externas, que le permitan aprovechar de mejor manera el potencial atractor que presenta junto con las fortalezas de ubicación y articulador comercial. Esto deberá realizarse atendiendo los intereses de los pobladores involucrándolos en los procesos de planeamiento, diseño y ejecución.

a) Proyecto representativo: renovación de expresiones de identidad colectiva, se trata de la concepción del bien común centrada en la construcción popular de diseños participativos que permitan legitimar el espacio público.

\section{Lineamiento 2. Manejo adecuado de la oferta de los ecosistemas naturales y las demandas sociales}

Lugares con identidad y percepción: estos deberían ser reorganizables, memorizables, revitalizables, que atraigan la atención diferenciándose de otros lugares. 
La identidad depende del conocimiento del observador y puede transmitirse indirectamente mediante símbolos verbales.

a) Proyecto representativo: Ruta del Arriero y Agro-comerciante, se centra en la valoración y rescate de las antiguas rutas y puntos de encuentro de carácter patrimonial, histórico y cultural para estructurar un turismo planificado que pueda integrarse con otras ciudades que hacen parte de la ruta del café.

Impulso al desarrollo empresarial: mitigación de los impactos del proceso de globalización en las actividades económicas; se busca la protección de los recursos, el empleo local y una economía popular, centrada en el interés general y en el trabajo como bien social.

a) Proyecto representativo: creación de Factorías Comunitarias, funcionando a nivel "micro-local" (comunidad o barrio) que permitan coordinar diferentes líneas de producción micro-empresarial con el fin de multiplicar la gama de ofertas y de bienes de servicio a la comunidad.

\section{Lineamiento 3. Calidad y accesibilidad del paisaje}

Paisaje organizado: es necesario técnicamente que sus partes armonicen, pero que no sean sólo funcionales sino también coherentes para los sentidos, que su imagen visual sea congruente con su vida y utilización.

a) Proyecto representativo: Rescate de la Malla Verde, para la generación de vínculos con la percepción de bienestar y posibilidad de acceso a un medio ambiente saludable que se observa en la presencia de una malla verde en buenas condiciones y adecuada para las determinantes climáticas.

Aseguramiento de la calidad de la oferta territorial rur-urbana: se hace necesario emprender acciones que permitan articular adecuadamente la estructura ecológica principal y la realidad urbanística del sub-tramo con el municipio. En otras palabras, se establece una relación de doble vía entre el hecho urbano y la estructura físico-ambiental que le brinda soporte, en el marco de la cual se gesta el concepto de paisaje. 
a) Proyecto representativo: Feria al Parque, se refiere a generar encuentros de comercialización y reproducción económico-cultural, que refuercen los lazos de socialización rur-urbana.

\section{Conclusiones}

La recuperación histórica de una ciudad es vital porque le permite reencontrarse a sí misma, a partir de un legado y un esfuerzo conjunto que marcaron un hito en dicho momento y forjaron un devenir; tener presente las tradiciones no sólo es apostarle a lo visible, también es visibilizar lo invisible.

El territorio como trama del desarrollo local está supeditado a los poderes políticos, la mayoría de las veces dicha planificación o forma de hacer ciudad se basa en un diagnóstico descontextualizado imponiéndose un trabajo de escritorio, afortunadamente durante estos últimos años se ha despertado de ese letargo y se comienzan a hacer trabajos que incluyen la comunidad de una manera más sólida, pero aún es insuficiente porque a las comunidades también hay que despertarlas de su propio letargo y facultarlas para que puedan ejercer su derecho de ciudadanía.

Existe toda una plataforma jurídica y de gestión desde hace ya varias décadas, e inclusive hay instrumentos para la participación ciudadana, pero la dificultad de operacionalizar y plasmar dichos instrumentos en el territorio depende de la habilidad de sus instituciones, no sólo en un camino físico-espacial en cuanto tal, sino a través de un seguimiento socio-territorial de la pertinencia, que pierde vigencia entre la formulación concertada y la aplicabilidad carente de celeridad y concreción.

El hábitat como esa relación estrecha entre el ser humano y su entorno, permite que exista un sentido de arraigo y apropiación por el espacio que se ocupa, aunque dicha relación es tan frágil que se puede perder en algún momento, por las constantes fluctuaciones que tiene la sociedad con el Estado, depende de este recuperar esos sentidos e imaginarios que antes permitieron crear lazos de convivencia y autonomía en busca de un nuevo desarrollo del lugar y un quehacer de la sociedad. 


\section{Referencias}

COLOMBIA. Asociación Nacional de Industriales. Cuadragésima Asamblea Seccional Anual de Afiliados a la Asociación Nacional de Industriales-ANDI, seccional Risaralda. Pereira, 2001.

ASOCIACIÓN NACIONAL DE INDUSTRIALES (Colombia). Cuadragésima Asamblea Seccional Anual de Afiliados a la Asociación Nacional de Industriales ANDI, seccional Risaralda. Pereira, 2001.

COLOMBIA. Corporación Autónoma Regional de Risaralda (CARDER) y Universidad Católica Popular de Risaralda (UCPR). Facultad de Artes. Programa de Arquitectura y Diseño Industrial. Convenio 069. Pereira: CARDER, 2008.

COLOMBIA. Corporación Autónoma Regional de Risaralda (CARDER). Agenda ambiental del municipio de Pereira. Pereira: CARDER, 2007.

COLOMBIA; Planeación Municipal de Pereira; UNIVERSIDAD CATÓLICA POPULAR DE RISARALDA. Plan local de ordenamiento del Centro Poblado la Bella. Pereira: Alcaldía Municipal, 2002.

CUBILLOS, J. Arqueología del Valle del Río Cauca: asentamientos prehispánicos en la suela Plana del Río Cauca. Bogotá: Banco de la República; Fundación de Investigaciones Arqueológicas Nacionales, 1984.

DÍAZ, C. Metodología interdisciplinaria desde el estudio de la problemática ambiental del tramo urbano de la Cuenca del Río Consota: hacia el fortalecimiento de la gestión ambiental local. 2007, 199 f. Disertación ( Maestría in Ingeniería y Arquitectura) Universidad Nacional de Manizales, Facultad de Ingeniería y Arquitectura, Manizales, 2007.

DOUROJEANNI, A. Procedimientos de gestión para el desarrollo sustentable: aplicados a microrregiones y cuencas. Santiago de Chile, 1994.

DUQUE, L.; FRIEDE, J.; JARAMILLO, J. Historia de Pereira. 1 ed. Bogotá: Club Rotario de Pereria, 1963.

DURÁN, L. Interfases ambientales para la construcción de hábitat colectivo: Cuenca media del Río Otún, Colombia. Saarbrücken: Editorial Académica Española, 2012. 
GIL, A.; ALVAREZ, D. Caracterización del mercado de fabricación y distribución de fajas en las ciudades de: Bogotá, Medellín, Envigado, Itagüí, Cali, Tuluá, Yumbo, Armenia, Pereira, Manizales y Barranquilla. 2011, 198 f. Monografía (Graduacion in Ingeniería Industrial) Universidad Tecnológica de Pereira, Facultad de Ingeniería Industrial, Pereira, 2011.

GIRALDO, T. Propuesta de gestión para el desarrollo de una región metropolitana en la ecorregión eje cafetero. 2007, 156 f. Disertación (Maestría in Arquitectura e Ingeniería) Universidad Nacional de Colombia, Facultad de Arquitectura e Ingeniería. Manizales, 2007.

GONZÁLEZ C. et al. Cuando el río vuelve: Recuperación ambiental y paisajística del río Consota de Pereira. Pereira: Universidad Católica Popular del Risaralda, 2011.

ISAZA, J. Conurbación y desarrollo sustentable: Una estrategia de intervención para la integración regional. Caso: Primer anillo metropolitano Bogotá-Sabana de Occidente. 2008, 320 f. Disertación (Maestría in Arquitectura y Diseño) Pontificia Universidad Javeriana, Facultad de Arquitectura y Diseño, Bogotá, 2008.

LEFF, E. La complejidad ambiental. México: Siglo XXI, 2000.

LÓPEZ, C; CANO, C; MORA, L. Patrimonio Arqueológico y paisajes culturales: La presencia humana milenaria en el Departamento de Risaralda. In: U.C.P.R., U.T.P, CARDER. Paisaje Cultural Cafetero. Pereira, 2008. p. 83-109.

NOGUERA, A. Augusto Angel Maya: poeta-filósofo del pensamiento ambiental latinoamericano. ISEE Publicación Ocasional. Sección Filosofía Ambiental Sudamericana, n. 6, 2009.

PESCI, R et al. Proyectar la sustentabilidad: enfoque y metodología de FLACAM para proyectos de sustentabilidad. La Plata: CEPA, 2007.

PESCI, R. Del titanic al velero: La vida como proyecto. Argentina: CEPA, 2000.

ROJAS, B. La urbanización y la dinámica poblacional en la generación de condiciones de riesgo en Pereira: Una evaluación ambiental histórica 1950-2000. 2003, 270 f. Monografía (graduación in Ciencias Ambientales) - Universidad Tecnológica de Pereira, Facultad de Ciencias Ambientales, Pereira, 2003. ,

VANEGAS, J. Desarrollo físico espacial y social municipio de Pereira. Secretaría Municipal de Pereira. Departamento de Información y Sistemas. Pereira, 1997. 
Interfases territoriales y construcción histórico ambiental del hábitat: tramo urbano Rio Otún, Pereira-

Colombia

Luisa Fernanda Durán Montes, César Andrés Alzate Hoyos, Samuel Darío Guzmán López

Recebido em: 10/03/2015 Aprovado em: 30/04/2015

Universidade do Estado de Santa Catarina - UDESC

Centro de Ciências Humanas e da Educação - FAED

Revista PerCursos

Volume 16 - Número 30 - Ano 2015 revistapercursos@gmail.com 